\title{
Delayed Onset of Transversus Abdominus in Long-Standing Groin Pain
}

\author{
SALLIE M. COWAN ${ }^{1}$, ANTHONY G. SCHACHE ${ }^{2}$, PETER BRUKNER ${ }^{1}$, KIM L. BENNELL $^{1}$, PAUL W. HODGES ${ }^{3}$, \\ PAUL COBURN $^{4}$, and KAY M. CROSSLEY ${ }^{1}$ \\ ${ }^{I}$ The Centre for Sports Medicine and Research and Education, School of Physiotherapy, The University of Melbourne, \\ Melbourne, AUSTRALIA; ${ }^{2}$ Murdoch Children's Research Institute and Hugh Williamson Gait Laboratory, Royal \\ Children's Hospital, Parkville, Victoria, AUSTRALIA; ${ }^{3}$ Department of Physiotherapy, The University of Queensland, \\ Queensland, AUSTRALIA; and ${ }^{4}$ Lifecare Physotherapy South Morang, Victoria, AUSTRALIA
}

\begin{abstract}
COWAN, S. M., A. G. SCHACHE, P. BRUKNER, K. L. BENNELL, P. W. HODGES, P. COBURN, and K. M. CROSSLEY. Delayed Onset of Transversus Abdominus in Long-Standing Groin Pain. Med. Sci. Sports Exerc., Vol. 36, No. 12, pp. 2040-2045, 2004. Long-standing groin pain is a persistent problem that is commonly difficult to rehabilitate. Theoretical rationale indicates a relationship between the motor control of the pelvis and long-standing groin pain; however, this link has not been investigated. Purpose: The current experiment aimed to evaluate motor control of the abdominal muscles in a group of Australian football players with and without long-standing groin pain. Methods: Ten participants with long-standing groin pain and 12 asymptomatic controls were recruited for the study. Participants were elite or subelite Australian football players. Fine-wire and surface electromyography electrodes were used to record the activity of the selected abdominal and leg muscles during a visual choice reaction-time task (active straight leg raising). Results: When the asymptomatic controls completed the active straight leg raise (ASLR) task, the transversus abdominus contracted in a feed-forward manner. However, when individuals with long-standing groin pain completed the ASLR task, the onset of transversus abdominus was delayed $(P<0.05)$ compared with the control group. There were no differences between groups for the onset of activity of internal oblique, external oblique, and rectus abdominus (all $P>0.05$ ). Conclusions: The finding that the onset of transversus abdominus is delayed in individuals with long-standing groin pain is important, as it demonstrates an association between long-standing groin pain and transversus abdominus activation. Key Words: OSTEITIS PUBIS, ELECTROMYOGRAPHY, ABDOMINAL MUSCLE, AUSTRALIAN FOOTBALL, SYMPHYSIS JOINT
\end{abstract}

$\mathrm{G}$ roin pain is a common complaint in many sporting populations, particularly those that involve kicking and rapid change of direction movements. Relatively high incidence rates have been well documented in sports such as soccer (4) and hockey (5). In Australian football, groin pain is the second most common complaint, accounting for around 14 matches missed per club per season (19).

Australian football is a unique sport played outdoors on natural-grass playing fields that vary in length from 140 to $180 \mathrm{~m}$. Ball progression is primarily achieved via punt kicking. The season consists of 22 matches per team, with teams playing $1 \times \mathrm{wk}^{-1}$. Each team has 18 players on the field and 4 players on an interchange bench. Games are

Address for correspondence: Dr. Sallie Cowan, The Centre for Sports Medicine Research and Education, School of Physiotherapy, The University of Melbourne, 200 Berkeley St., Carlton, Vic, 3010, Australia; E-mail: s.cowan@unimelb.edu.au.

Submitted for publication January 2004.

Accepted for publication July 2004.

0195-9131/04/3612-2040

MEDICINE \& SCIENCE IN SPORTS \& EXERCISE E $_{\circledast}$

Copyright (C) 2004 by the American College of Sports Medicine

DOI: $10.1249 / 01 . M S S .0000147587 .81762 .44$ played over four quarters lasting $20 \mathrm{~min}$ each, with additional time for breaks in play (18).

Groin pain in athletes is frequently a difficult problem to resolve. It can be attributed to a variety of different disorders, few of which have unambiguous diagnostic criteria that are uniformly accepted (12). Furthermore, groin pain is often diffuse, difficult to interpret, and prone to chronicity (20).

From a clinical perspective, the greatest challenge is provided by groin pain that becomes chronic. Although the pathophysiology of chronic groin pain is not known, a common feature is overuse or repetitive stress on the tissues (hard and/or soft) of the groin region. Imaging typically reveals features indicative of stress to the pubic symphysis and adjacent pubic bone (25). These structures may therefore be a potential source of symptoms in long-standing groin pain.

Flat longitudinally orientated joints are mechanically most vulnerable to shear forces (23). Consistent with this, biomechanical studies have demonstrated that the pubic symphysis displays its greatest mobility when exposed to shear forces (26-28). The anterior pelvic ring therefore requires mechanisms with which to stabilize against such forces. Structures orientated perpendicular to the pubic symphysis joint surface provide one mechanism capable of providing this stability. The thick inferior pubic (arcuate) ligament is the main passive structure providing resistance against shear forces, as the other pubic ligaments are thin, 
and likely to contribute little to the mechanical stability of the joint (6). Dynamic structures providing resistance against shear forces are likely to constitute muscles capable of compressing the pelvic ring anteriorly. Transversely orientated deep abdominal muscles such as the lower fibers of transversus abdominis and internal oblique appear to be mechanically most capable of performing this function (22).

The lower fibers of transversus abdominis and internal oblique arch medially and downward to merge into a flat aponeurosis known as the falx inguinalis (conjoint tendon). The falx inguinalis, together with the underlying fibers of the rectus abdominis, attach to the superior aspect of the pubic bone immediately adjacent to the symphysis on both sides (15). As the lower fibers of transversus abdominis and internal oblique originate from the anterior iliac spine and inguinal ligament, contraction of these muscles will tend to approximate the anterior iliac spines, thus compressing the superior edge of the pubic symphysis and distracting the inferior edge. If this action is also coordinated with a contraction of the pelvic floor muscles from below, a net compressive effect on the pubic symphysis is theoretically achievable.

Given the potential stabilizing role for the transversely orientated abdominal muscles at the anterior pelvic ring, optimal control of these muscles may be a relevant consideration in the context of long-standing groin pain. In support of this theoretical rationale, other studies investigating the recruitment patterns of the transversely orientated abdominal muscles in subjects with low back pain (LBP) (10) and sacroiliac joint pain $(13,17)$ have found motor-control deficits of these muscles, manifested by changes in the timing of activity. It is therefore feasible that a motor-control deficit may also occur in association with long-standing groin pain. However, this has not been investigated. The aim of the current experiment is to evaluate in a group of Australian football players: (a) the normal motor control of the transversely orientated abdominal muscles associated with a pelvic perturbation, and (b) whether there is an alteration in the motor control of the transversely orientated abdominal muscles in the presence of long-standing groin pain.

\section{METHODS}

\section{Subjects}

Ten participants with long-standing groin pain (defined as groin pain present for greater than $6 \mathrm{wk}$ ) and 12 asymptomatic participants were recruited for the study. Participants were elite or subelite Australian football players (see Table 1 for definition), and were recruited from health professionals, advertisements, and media in Victoria, Australia.

The inclusion/exclusion criteria for the long-standing groin pain group and the control subjects are detailed in Table 1. These criteria were based on criteria used in a recent randomized controlled trial (12)

The mean (SD) age, height, weight, and number of games played of the long-standing groin pain subjects were 26 (7) yr, $180.7(5.7) \mathrm{cm}, 78.1$ (8.4) kg, and 90 (74) games. The
TABLE 1. Inclusion/exclusion criteria.

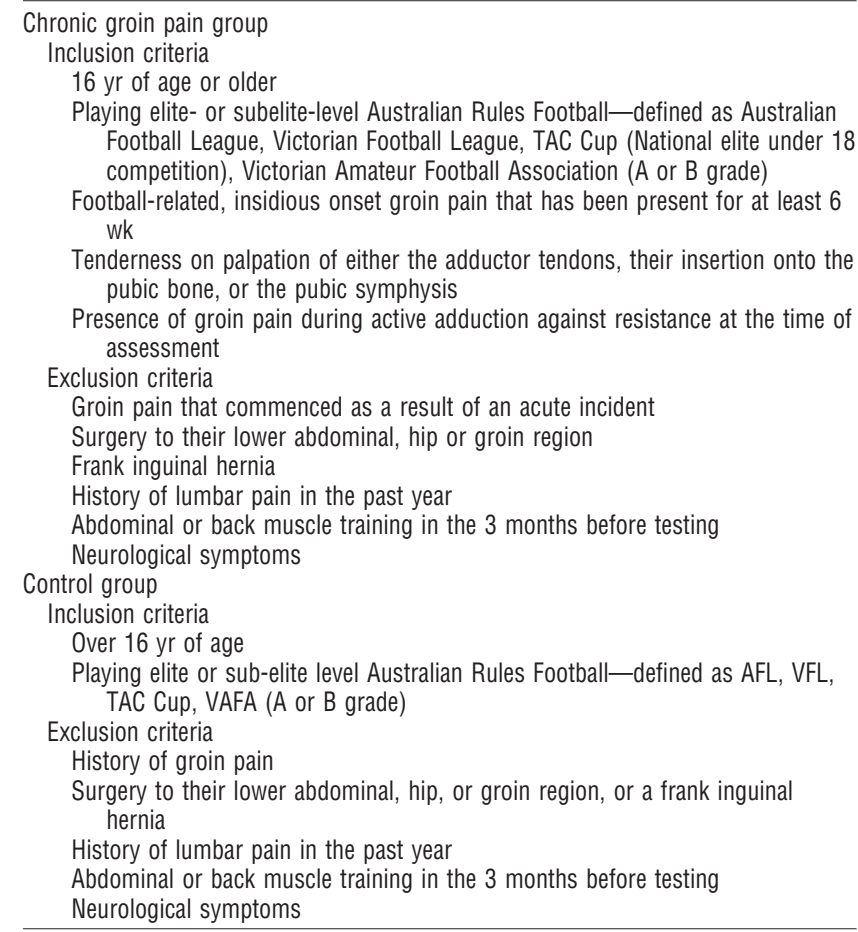

mean (SD) age, height, weight, and number of games played of the control subjects were 25 (6) yr, $176.5(7.9) \mathrm{cm}, 76.8$ (11.3) kg, and 110 (92) games. There were no significant differences between groups for these variables assessed using independent $t$-tests.

The study was approved by the University of Melbourne Human Research Ethics Committee. All subjects provided written informed consent.

\section{Electromyographic Recordings}

EMG activity of transversus abdominus (TrA) (lower portion), obliquus internus (OI), and externus (OE) were recorded using bipolar intramuscular electrodes fabricated from Teflon-coated stainless steel wire $75 \mu \mathrm{m}$ in diameter (AM Systems, Carlsborg, WA). One millimeter of insulation was removed from the cut ends of the wire, and the tips were bent back approximately $1-2 \mathrm{~mm}$ from the end to form a hook. The electrodes were threaded into a hypodermic needle $(0.70 \times 38 \mathrm{~mm})$ and sterilized.

The electrodes were inserted into TrA, OI, and OE under the guidance of real-time ultrasound imaging $(7.5-\mathrm{MHz}$ curved linear array transducer, Dornier Performa, Acoustic Imaging Technologies Corp., Phoenix, AZ) (8). Electrodes were inserted on the symptomatic side (or most severe side if bilateral) and on the right side for control subjects. The fine-wire electrodes for TrA and OI were inserted below the level of the anterior superior iliac spines to ensure that the lower fibers of the muscles were recorded.

EMG activity was recorded using pairs of surface $\mathrm{Ag}$ / $\mathrm{AgCl}$ electrodes (Graphics Control Corporation, c/o Medical Equipment Services PTY.LTD, Richmond, Australia) placed over the muscle bellies of rectus femoris (RF) 
and rectus abdominus (RA) with an interelectrode distance of $22 \mathrm{~mm}$.

EMG data were preamplified $(10 \times)$, bandpass filtered between 20 and $1000 \mathrm{~Hz}$, sampled at $2000 \mathrm{~Hz}$, and 12-bit A-D converted (Associative Measurement Pty, Ltd, North Ryde, N.S.W., Australia). All data were exported for analysis with IGOR Pro (Igor Pro 4, Wavemetrics, Inc., Lake Oswego, OR).

\section{Procedure}

Active straight leg raise (ASLR) task. To create a known mechanical perturbation to the pelvic ring, the ASLR task was used. This task has been shown to have a strong correlation with the mobility of the pelvic joints in individuals with pregnancy-related pelvic girdle pain (16). Subjects lay in supine positions with pillows under their heads. They were instructed to lift their straight leg off the bed to approximately $45^{\circ}$ (ASLR), or to push their straight leg into the bed as quickly as possible in response to a light. The direction of movement was indicated by a differently colored light in a choice reaction-time task. The onset of movement was measured using a standard pressure biofeedback unit (Chattanooga Group, Hornsby, Sydney, Australia) sampled at $2000 \mathrm{~Hz}$. Subjects were instructed not to keep their leg in the test position at the completion of the task, nor to attempt to return to the exact initial position. Subjects were instructed to remain relaxed between trials. Data were collected for 10 repetitions of the ASLR task, and data were not analyzed for the task in which the leg was pressed directly into the bed, as this task was not considered to cause a significant perturbation to the pelvic ring. This was included to make the task a choice reaction-time task, thus reducing the potential for the subject to prepare for the movement.

Pain and disability measures. Patients rated their worst and usual pain in the last week and their worst groin pain during their most common aggravating activities on a 10-cm VAS. Participants rated the functional limitation of their groin pain of a five-point Likert scale ranging from none ("groin pain doesn't limit your activities at all") to completely disabling ("you can do very little activity due to your groin pain").

Data analysis. The onset of EMG was identified visually from the raw data as the point at which EMG increased above the baseline activity. To remove the possibility of observer bias, traces were displayed individually, with no reference to group. There was no reference to muscle or trial number, to any parameter of movement, or to EMG of another muscle. To assess any interobserver variance, onset times for each muscle were assessed by two separate examiners using data from 15 trials in two participants. There were no significant differences between the two examiners $(P=0.42)$, and the two scores were closely matched with a mean absolute difference of $1.4 \mathrm{~ms}$ with a standard deviation of $2.6 \mathrm{~ms}$.

The relative latencies between the onset of the rectus femoris and that of the abdominal muscles were used for analysis. Trials were excluded if the onset of abdominal muscle EMG occurred more than $200 \mathrm{~ms}$ before or after that of RF (as this is likely to indicate that the abdominal response was unrelated to the task). Fewer than $5 \%$ of trials were excluded on this basis.

\section{Statistical Analysis}

Data were analyzed for normality and homogeneity of variance. Individual one-group $t$-tests were used to compare the latency of muscle onset with the prime-mover RF (onset $=0$ ) in both the control and the long-standing groin pain groups.

Unpaired $t$-tests were used to compare muscle onset, reaction time (onset of RF relative to visual stimulus to commence task), and onset of movement between the control and long-standing groin pain participants. The alpha level was set at 0.05 .

Univariate correlations were sought in the long-standing groin pain group between EMG onset, symptom duration, pain, age, height, and weight.

\section{RESULTS}

Normal response. When participants with no history of groin pain completed the ASLR task, the onset of EMG of $\operatorname{TrA}$, IO, and RA muscles preceded that of RF, and the onset of EMG of OE occurred after that of RF (Fig. 1). However, one-group $t$-tests demonstrated that only the onset of TrA EMG occurred significantly before that of the primemover RF $(P=0.024)$.

Long-standing groin pain subjects. The pain and disability characteristics of the long-standing groin pain group are detailed in Table 2; long-standing groin pain participants had a worst pain (SD) during their most aggravating activity of 6.5 (1.9) measured on a 10-cm VAS. Most
A

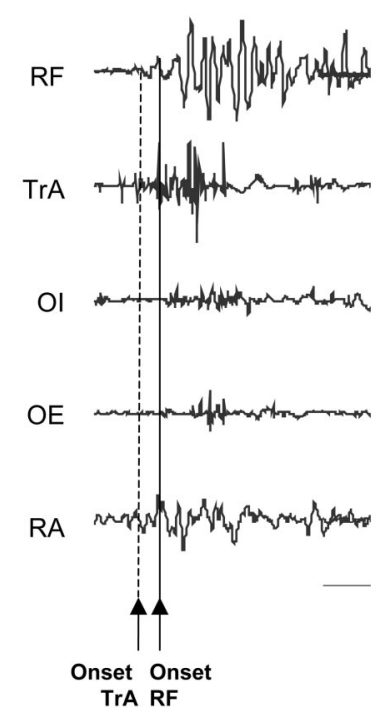

B

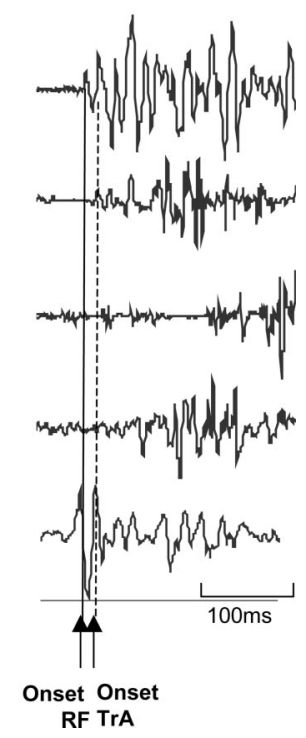

FIGURE 1-Representative raw EMG data for an asymptomatic individual (A) and an individual with long-standing groin pain (B) in association with the active straight leg raise task (ASLR). 
TABLE 2. Groin pain (measured on a 10-cm VAS) and functional limitation (measured on a five-point Likert scale) of the chronic groin pain group.

\begin{tabular}{lcl}
\hline & Mean (SD) & Range \\
\hline Worst pain in the last week & $5.5(2)$ & $3-9$ \\
Average pain in the last week & $2.5(2)$ & $0-6$ \\
Pain on most aggravating activity & $6.5(2)$ & $3-9$ \\
Pain on running & $6.0(2)$ & $2-8$ \\
Functional limitation & $3(0.5)$ & $2-4$ \\
Symptom duration (wk) & $56(82)$ & $6-260$ \\
\hline
\end{tabular}

common aggravating activities included kicking, sprinting, and changing directions while running. The majority of long-standing groin pain group $(N=7)$ complained of bilateral groin pain.

When participants in the long-standing groin pain group completed the ASLR task, there were no significant differences between the onsets of the abdominal muscles and that of the prime mover for the task RF (all $P>0.28$ ). Representative EMG data for a participant in the long-standing groin pain group is detailed in Figure 1.

Comparison between long-standing groin pain and control participants. Figure 2 illustrates the means and standard errors of the EMG onset of muscle activity of the abdominal muscles with respect to the onset of RF EMG, the prime mover of the ASLR task. Unpaired $t$-tests indicated that there was a significant difference in the onset of TrA between the control and long-standing groin pain groups $(P<0.05)$, with the onset of TrA occurring later with respect to RF in the long-standing groin pain group. There were no significant differences in the onset of activity of IO, OE, and RA between the control and long-standing groin pain groups (all $P>0.05$ ). The onset of movement occurred later in the long-standing groin pain group $(P<0.03)$, but there

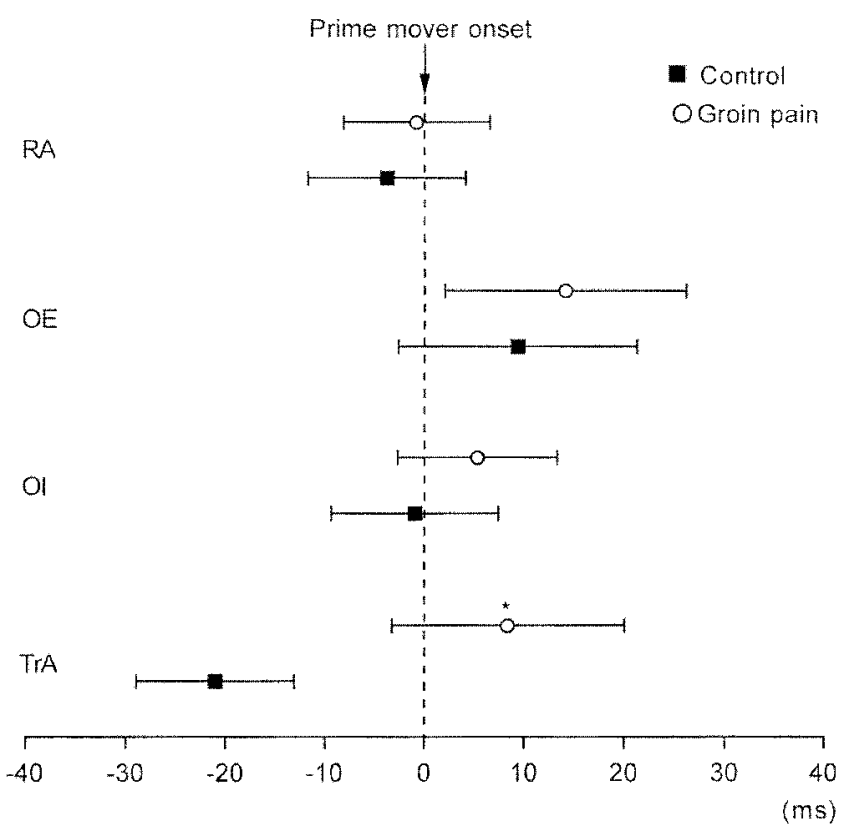

FIGURE 2-Mean EMG onset of the abdominal muscles in the control and long-standing groin pain groups for the ASLR task. All mean onsets are aligned to the onset of the prime mover (RF) at zero, denoted by the dashed line. Note the significant delay of TrA EMG in the long-standing groin pain group. Standard error bars are shown; $* P<0.05$.

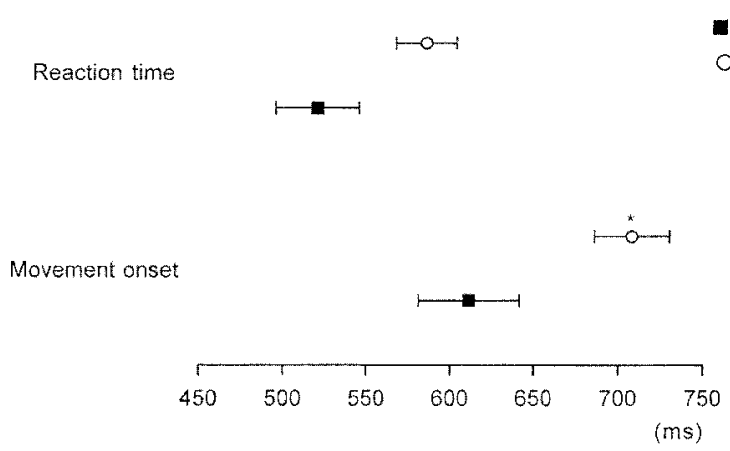

FIGURE 3-Mean (standard error) onset of movement (onset of pressure change) and reaction time (onset of the prime-mover $R F$ ) in the long-standing groin pain group and the control group; $* P<0.03$.

was no difference in the onset of the prime-mover RF (i.e., reaction time) between groups $(P=0.055)$ (Fig. 3).

Correlations between pain, disability, and EMG onset. Participants with a longer history of long-standing groin pain tended to have greater average pain during the last week ( $\mathrm{r}=0.712, P=0.021)$. There were no significant correlations between height, weight, age, pain, and disability measures and onset of EMG of any of the muscles.

\section{DISCUSSION}

The results of this study demonstrate that the contraction of TrA occurs as part of a feed-forward response when a voluntary movement that is hypothesized to perturb the pelvic ring is performed. However, when the same ASLR movement is performed in individuals with long-standing groin pain, the onset of $\operatorname{Tr} A$ is delayed when compared with the normal situation. It is hypothesized that this change in coordination of TrA muscle activity may leave the pelvic ring unprotected from reactive forces, resulting from movement.

Feed-forward contraction of the abdominal muscles in asymptomatic individuals. Based on biomechanics (26-28), anatomy (22), and previous studies (8), the finding that $\operatorname{TrA}$ contracted in a feed-forward manner in association with a perturbation of the pelvic ring was not surprising. Hodges and Richardson (8) demonstrated feedforward activation of the abdominal muscles in association with lower-limb movement in a similar task with the participants standing. It is well established that the CNS deals with perturbations to the spine by contraction of the muscles of the trunk either before or shortly after the onset of EMG activity of the muscle responsible for movement of the limb $(1,8)$. It follows that the CNS would deal with similar perturbations to the pelvic ring in the same feed-forward manner.

The onset of transversus abdominus is delayed in individuals with long-standing groin pain. The finding that the onset of TrA was delayed in individuals with long-standing groin pain is consistent with previous studies of LBP populations (9). In association with upper-limb movement in people with LBP, the onset of TrA was found to occur more than $50 \mathrm{~ms}$ after the onset of EMG of the 
prime mover of the limb (9). This indicates that the contraction of $\operatorname{Tr}$ A may have been mediated by afferent input from the movement and not as a component of the feedforward postural response (9). The onset of TrA in our long-standing groin pain population, although delayed, occurred less than $50 \mathrm{~ms}$ after the onset of the prime mover of the limb and, therefore, still occurred within the feed-forward criteria. Thus, with a perturbation to the pelvic ring caused by an ASLR, the contraction of TrA must still be preprogrammed by the CNS, although delayed. This is consistent with studies investigating the effect of a lower-limb perturbation in an LBP population (9).

The findings are also consistent with O'Sullivan et al. (17), who investigated motor-control strategies of individuals with a diagnosis of sacroiliac joint pain. O'Sullivan et al. (17) used the ASLR task to perturb the pelvic ring, and found altered motor-control strategies in individuals with sacroiliac joint pain. Although these authors did not specifically measure TrA EMG activity, their finding that aberrant movement of the pelvic floor was associated with sacroiliac joint pain supports our findings of altered neuromotor control of the lumbo-pelvic system in response to a perturbation invoked by the ASLR task in individuals with long-standing groin pain.

Due to the cross-sectional design of our study, it is not possible to establish whether the delay in onset of EMG of TrA in the long-standing groin pain population was present before the onset of pain and could thus be seen as a causative factor or, conversely, if the timing alteration has occurred as a result of the presence of pain and dysfunction associated with the condition. It is also not possible to establish the mechanism of delay. There are a number of potential mechanisms for the delay, including reflex inhibition due to effusion $(2,24)$, ligament stretch, or capsular compression (3). At the sacroiliac joint, it has been demonstrated in a porcine model that stimulation of joint afferents affects lumbar muscle function (14). It is also possible that the presence of pain resulted in the aberrant motor control of TrA. Hodges et al. (7) demonstrated that acute experimentally induced pain consistently impaired the feed-forward response of TrA. Furthermore, it is important to note that the consequence of such a delay in $\operatorname{TrA}$ activity cannot be established from the present study.

The finding of a delay in TrA in long-standing groin pain subjects is also important in that it provides support for current physiotherapy management of long-standing groin pain in Australia. The current treatment regime used by physiotherapists at most Australian Football League clubs is

\section{REFERENCES}

1. Aruin, A. S., and M. Latash. Directional specificity of postural muscles in feed-forward postural reactions during fast voluntary movement. Exp. Brain Res. 103:323-332, 1995.

2. de Andrade, J., C. Grant, and A. Dixon. A joint distension and reflex muscle inhibition in the knee. J. Bone Joint Surg. 47A:313322, 1965.

3. Eкholm, J., G. Eklund, and S. Skoglund. On reflex effects from knee joint of cats. Acta Physiol. Scand. 50:157-174, 1960. based on the work of Richardson et al. (21), and has been adapted to long-standing groin pain by Hogan (11). This program is based on restoring neuromotor control of the $\operatorname{Tr} A$ with the premise that it contributes to the stabilization of the pelvic ring.

Limitations. There are a number of limitations that require consideration. First, a nonfunctional task was used to perturb the pelvic ring, limiting the generalization of the motor-control deficit found in this study to more functional activities. However, the task was specifically chosen because it had been demonstrated to perturb the pelvic ring (16); by performing the task in a supine position, the supporting the role of the abdominals in single-leg balance was removed. Thus, further study could include functional tasks such as kicking.

Second, although the ASLR task used was chosen because it perturbs the pelvic ring, this task also perturbs the lumbar spine (17). Thus, we can not be certain that the finding of a delay in the activation of TrA was associated with the perturbation of the pelvic ring, as the finding might have been due to a perturbation of the lumbar spine produced by the ASLR task (indeed, as both the lumbar spine and pelvic ring are perturbed, it is likely that the observed response occurs to control both segments). This is a difficult problem, as the anatomy/biomechanics of the lumbo-pelvic region dictate that a task that specifically perturbs an isolated segment, such as the pubic symphysis, would be virtually impossible to design.

Additional limitations include the fact that only the symptomatic side was measured. Further study should include assessment of muscle activation on the asymptomatic (or less symptomatic side for participants with bilateral groin pain), as it is possible/likely that the delay in $\operatorname{TrA}$ activation occurs bilaterally. Also of importance would be to establish whether physiotherapy treatment commonly used for longstanding groin pain (see above) is able to alter neuromotor control of $\operatorname{TrA}$ in these individuals.

Lastly, also of note is the variable temporal response of the abdominal muscles accompanying limb movement in seemingly constant conditions. There are a number of possible explanations for this variation, including variability between subjects, and variations in placement of fine-wire electrodes. However, the degree of variability for the majority of the muscles investigated is comparable to previous studies investigating abdominal activity in $\operatorname{LBP}(9,10)$.

This study was funded by a grant from the Australian Football League.

4. Ekstrand, J., and J. Hiding. The incidence and differential diagnosis of acute groin injuries in male soccer players. Scand. J. Med. Sci. Sports 9:98-103, 1999.

5. Emery, C. A., W. Meeuwise, and J. W. Powell. Groin and abdominal strain injuries in the national hockey league. Clin. J. Sport Med. 9:151-156, 1999.

6. Gamble, J. G., S. C. Simmons, and M. Freedman. The symphysis pubis: anatomic and pathologic considerations. Clin. Orthop. Relat. Res. 203:261-272, 1986. 
7. Hodges, P. W., G. L. Moseley, A. Gabrielsson, and S. C. GanDEVIA. Experimental muscle pain changes feedforward postural responses of the trunk muscles. Exp. Brain Res. 151:262-271, 2003.

8. Hodges, P. W., and C. A. Richardson. Contraction of the abdominal muscles associated with movement of the lower limb. Phys. Ther. 77:132-142, 1997.

9. Hodges, P. W., and C. A. Richardson. Delayed postural contraction of transversus abdominis in low back pain. J. Spinal Disord. 11:46-56, 1998.

10. Hodges, P. W., and C. A. Richardson. Inefficient muscular stabilization of the lumbar spine associated with low back pain. Spine 21:2640-2650, 1996.

11. Hogan, A. A rehabilitation model for pubic symphysis injuries. In: Australian Conference of Science and Medicine in Sport, Adelaide, 1998, p. 143.

12. Holmich, P., P. Uhrskou, L. Ulnits, I. Kanstrup, M. Nielson, and A. BJERG. Effectiveness of active physical training as treatment for long-standing adductor-related groin pain in athletes: randomised trial. Lancet 353:439-443, 1999.

13. Hungerford, B., W. Gilleard, and P. W. Hodges. Evidence of altered lumbopelvic muscle recruitment in the presence of sacroiliac joint pain. Spine 28:1593-1600, 2003.

14. Indahl, A., A. Kaigle, O. Reikeras, and S. Holm. Sacroiliac joint involvement in activation of the porcine spine and gluteal musculature. J. Spinal Disord. 12:325-330, 1999.

15. McMinn, R. M. H. Last's Anatomy, 8th Ed. Edinburgh: Churchill Livingstone, 1990.

16. Mens, J. A., A. Vleeming, C. J. Snijders, H. J. Stam, and A. Z. GinAI. The active straight leg raising test and mobility of the pelvic joints. Eur. Spine J. 8:468-473, 1999.

17. O'Sullivan, P. B., D. J. Deales, J. A. Beetham, et al. Altered motor control strategies in subjects with sacroiliac joint pain during the active straight leg raise test. Spine 27:E1-E8, 2002.
18. ORChARD, J. Intrinsic and extrinsic risk factors for muscle strains in Australian Football. Am. J. Sports Med. 29:300-303, 2001.

19. Orchard, J., and H. Seward. Epidemiology of injuries in the Australian Football League, seasons 1997-2000. Br. J. Sports Med. 36:300-303, 2002.

20. Renstrom, P., and L. Peterson. Groin injuries in athletes. $B r . J$. Sports Med. 14:30-36, 1980.

21. Richardson, C. A., G. A. Jull, P. W. Hodges, and J. A. Hides. Therapeutic Exercise for Spinal Segmental Stabilization in Low Back Pain: Scientific Basis and Clinical Approach. Edinburgh: Churchill Livingstone, 1999.

22. Snijders, C., A. Vleeming, R. Stoeckart, J. A. Mens, and G. J. KLEINRENSINK. Biomechanical modeling of sacroiliac stability in different postures. Spine 9:419-432, 1995.

23. Snijders, C. J., A. Vleeming, and R. Stoeckart. Transfer of lumbosacral load to iliac bones and legs. Part I: Biomechanics of self-bracing of the sacroiliac joints and its significance for treatment and exercise. Clin. Biomech. 8:285-294, 1993.

24. Stokes, M., and A. Young. The contribution of reflex inhibition to arthrogenous muscle weakness. Clin. Sci. 67:7-14, 1984.

25. Verrall, G. M., J. P. Slavotinek, and G. T. Fon. Incidence of pubic bone marrow oedema in Australian Rules Footballers: relation to groin pain. Br. J. Sports Med. 35:28-33, 2001.

26. Walheim, G. G., S. Olerud, and T. Ribbe. Mobility of the pubic symphysis measurements by an electromechanical method. Acta Orthop. Scand. 55:203-208, 1984.

27. Walheim, G. G., and G. SLEvik. Mobility of the pubic symphysis measurements by an electromechanic method and a roentgen stereographammetric method. Clin. Orthop. Relat. Res. 191:129135, 1984.

28. WAng, M., and G. A. Daumas. Mechanical behaviour of the pubic symphysis: an in vitro study. In: Proceedings of the Canadian Society for Biomechanics, University of Calgary, 1994, pp. 303309. 